Check for updates

Cite this: RSC Adv., 2018, 8, 8671

Received 10th January 2018

Accepted 19th February 2018

DOI: $10.1039 / \mathrm{c} 8 \mathrm{ra} 00251 \mathrm{~g}$

rsc.li/rsc-advances

\section{A novel and actual mode for study of soil degradation and transportation of difenoconazole in a mango field}

\author{
Fangfang Zhao, (iD † abcd Jingkun Liu, $\uparrow^{\mathrm{e}}$ Defang Xie, ${ }^{\text {abcd }}$ Daizhu Lv ${ }^{\text {abcd }}$ \\ and Jinhui Luo*abcd
}

To supply actual data for assessing the potential threat from difenoconazole to the ecosystem, its practical environmental behaviors in a mango field were investigated through a novel mode. After optimization, a UPLC-MS/MS determination method with good accuracy and stability was developed that could be used for the residue determination. Difenoconazole residue was in situ sampled, and its degradation and transportation activity, which reflected the actual transfer characteristics in the natural environment, were researched. The results showed that the half-life of difenoconazole in the soil was 15.4 days, which may be accumulated in a year-round agricultural production system. The residue was detected in the rain settled underground, which showed that the residue transported gradually with the rainfall in vertical and horizontal directions. The results showed that difenoconazole would transport with the rainfall, although the process was slow. All the data showed that the soil ecosystem, and probably also the aquatic ecosystem, would be affected by difenoconazole residue.

\section{Introduction}

Difenoconazole, with the molecular structure as shown in Fig. 1, is a systemic triazole fungicide and acts by inhibiting demethylation during ergosterol synthesis. ${ }^{\mathbf{1} 2}$ It is now widely used for the protection of many crops, including vegetables, beans and fruits, ${ }^{3}$ for its excellent activity to control the fungus. In undeveloped areas, tropical areas of China and all over south Asia, mangoes are one of the most important economic

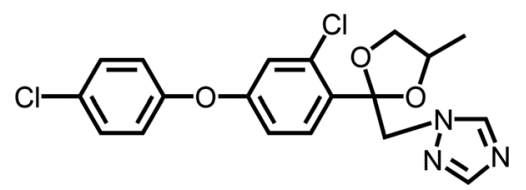

Fig. 1 The molecular structure of difenoconazole.

\footnotetext{
${ }^{a}$ Analysis \& Testing Center, Chinese Academy of Tropical Agricultural Sciences, Haikou, Hainan, 571101, China. E-mail: luocatas@21cn.com

${ }^{b}$ Laboratory of Quality \& Safety Risk Assessment for Tropical Products(Haikou), Ministry of Agriculture, Haikou, Hainan, 571101, China

'Hainan Provincial Key Laboratory of Quality and Safety for Tropical Fruits and Vegetables, Haikou, Hainan, 571101, China

${ }^{d}$ Quality Supervision and Inspection Center of Tropical Agro-Products, Haikou, Hainan, 571101, China

${ }^{e}$ Environment and Plant Protection Institute, Chinese Academy of Tropical Agricultural Sciences, Haikou, Hainan, 571101, China

$\dagger$ Co-first authors.
}

products, ${ }^{4}$ supporting thousands of farmers. Because of serious diseases, more and more fungicides are being used in tropical agriculture $^{5}$ to guarantee the mango yield and farmers' income, ${ }^{6,7}$ which poses a great threat not only to the safety of agro-products but also the farmland ecosystem, ${ }^{6-10}$ including soil, water and animals. ${ }^{11}$ For example, three types of neonicotinoid were banned by the EU for their severe damage to bee populations.

Soil and water, two of the most important environmental factors, are the basis of the ecosystem. Soil, the first and direct action subject of the pesticide in the environment, would be affected by the residue of the pesticide and the ecological factors in the soil, including the enzymes, microbes and animals, would also be threatened by them. Thus, the stability of the pesticide in the soil is closely related to soil health and ecological status. Difenoconazole is fat-soluble and stable in soil, which gives it a relatively long half-life. According to reports in the literature, ${ }^{12-14}$ the half-life of the difenoconazole is usually about 5-10 days and varies remarkably with the environmental conditions. Transportation is also one of the most important environmental behaviors of pesticides, which determines their distribution in agricultural ecology. The fungicide residue can be transported from plants and soil to rivers, grasses, forests and so on, which is also critical to ecological health. Difenoconazole in the soil can also be transport by rain and agricultural activity, resulting in effects on many aspects of agricultural ecology. Therefore, degradation and transportation are critical to assess the influence of difenoconazole on agricultural environment and ecology. 
Usually, the environmental behaviors of a pesticide are simulated in the lab, which does not represent the actual behavior of the pesticide in the field at all because this is the result of complex functions in the natural environment, including plant actions and photo-thermal effects. What is more serious in tropical farmland is that the heavy rain will accelerate the procedures. ${ }^{\mathbf{1 5 - 1 7}}$ It is important to know the actual degradation and transportation of the pesticide residue in the farmland, from which primary data on residue behaviors and a theoretical basis for measures of ecosystem protection could be provided.

Therefore, in situ field techniques and sampling methods are critical for monitoring the behaviors of pesticides in soil, especially the transportation from the soil to the water and that in the horizontal direction, which determines how the pesticide is transported in the whole ecosystem. However, it is difficult to conduct in situ techniques in farmland and there are also few reports ${ }^{18-21}$ about pesticide transport in the field environment, especially from the soil to the water. All of the experiments performed in the lab could not take the effects from the plants and other aspects into consideration, which greatly influence pesticide transport. Solid-phase micro-extraction (SPME) is the most widely used method in in situ field techniques, especially for gas and liquid samples. However, soil samples, a mixture of various matrices, are extremely complex for SPME to extract the trace amounts of the pesticide in soil or water. Soluble components in the samples would also be absorbed to the material in the SPME, lowering the extraction efficiency. Interferences from the matrix and small extraction capacity restrict the use of SPME for in situ study of pesticide residues in farmland. Furthermore, although the residue can be detected using sensitive equipment, its concentration will not be obtained for the unknown quantity of the samples. Thus, taking the environmental influences into consideration, an accurate, simple, valid and cheap in situ field method for the collection of the pesticide residue was developed. The actual characteristics of the degradation and transportation of difenoconazole residue in the mango field were clarified. Accurate and actual data for the pesticide residue would be helpful to assess its effects on the ecosystem, which is important for the protection of the agricultural environment and ecology.

\section{Materials and methods}

\section{Chemicals}

Standard difenoconazole was purchased from Dr Ehrenstorfer $\mathrm{GmbH}$ (Augsburg, Germany). HPLC-grade acetonitrile (ACN), methyl alcohol $(\mathrm{MeOH})$, ethyl acetate and acetone were obtained from Fisher (Thermo Fisher, USA). Water was purified through a Milli-Q apparatus (Ultima Duo 200 (COMBI), Balmann Tech, Daegu, Korea) prior to use in the experiment. Analytical-grade anhydrous magnesium sulfate $\left(\mathrm{MgSO}_{4}\right)$ and sodium chloride $(\mathrm{NaCl})$ were obtained from Junsei Chemicals Co., Ltd. (Tokyo, Japan). Primary secondary amine (PSA) was supplied by Agilent Technologies (Santa Clara, CA, USA). A stock standard solution $\left(1000 \mathrm{mg} \mathrm{\textrm {L } ^ { - 1 }}\right)$ of difenoconazole was prepared individually in $\mathrm{MeOH}$. An intermediate standard solution was prepared from the stock solution with further dilution in $\mathrm{MeOH}$. The working standard solution was prepared following serial dilution of the intermediate standard solution with the extract solution of the blank sample.

\section{Instruments}

An ultra-performance liquid chromatography (Acquity, Waters Corporation, Milford, MA) system coupled with tandem mass spectrometry (AB Sciex Corp., USA), an analytical weighing balance (AUY 220, Shimadzu, Kyoto, Japan), a homogenizer (IKA-T18) and a high speed freezing centrifuge (Himac CR 22N, Hitachi) were the main instruments in the experiment.

\section{Methods}

\section{Sampling method}

Experiments were conducted on Bayi farm in Danzhou, China $\left(109.336664^{\circ} \mathrm{E}, 19.461769^{\circ} \mathrm{N}\right)$. The soil was sandy latosol with a pH of 6.0 and organic matter content of $12.4 \mathrm{~g} \mathrm{~kg}^{-1}$. The mechanical composition of the soil showed that the percentage of the particles smaller than $0.01 \mathrm{~mm}$ was $32 \%$, indicating a moderate sandy latosol. Soil degradation of the pesticide was performed by spraying difenoconazole on the blank ground at a dose of $10 \mathrm{~g}$ (active ingredient, abbreviated as a.i.)/666 $\mathrm{m}^{2}$. The soil from the surface to a depth of $10 \mathrm{~cm}$ was sampled and wellmixed on days $0,1,3,7,14,21,28,42$ and 60 after the pesticide was sprayed. All the analyses were repeated three times. The mango trees grown in similar ground were used for the transportation experiment. The trees were spiked with the pesticide at $50 \mathrm{~g}$ (a.i.)/666 $\mathrm{m}^{2}$. To avoid the pesticide drifting to the soil outside the crown and influencing the results of the difenoconazole drift in the horizontal direction, the field outside of the crown was covered with plastic film when spraying. Bottles (500 $\mathrm{mL}$ ) covered with a screen mesh $(1 \mathrm{~mm})$ were uniformly buried underground in different spots (under and out of the crown) at three different depths of 10,20, and $30 \mathrm{~cm}$. Water samples at the same depth were distributed around one tree, showing two triangles under or out of the crown (Fig. 2). Another three test trees were used as the standby group in the experiment and prepared using the same method. At the same time, the blank group was treated with the same method except for spraying with water. The bottles from different spots (under and outside the crown) and depths $(10,20$ and $30 \mathrm{~cm})$ were taken out after

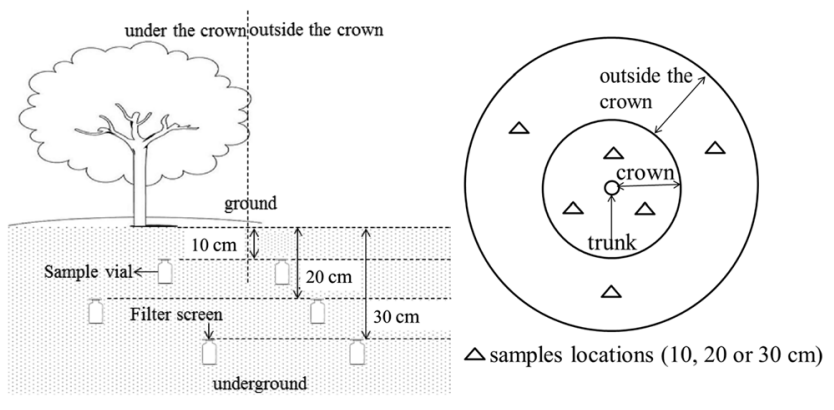

Fig. 2 Schematic diagram of the experimental design. 
each rainfall. Empty bottles were buried again in their original locations when the samples were removed. The water sampling was performed four times after it rained four times. All the samples, including the blank samples, had three replicates.

\section{Sample pretreatment method}

Water samples were only filtered using a membrane $(0.22 \mu \mathrm{m})$ and the soil samples were pretreated for the determination as follows: 10.0 grams of soil was centrifuged for $5 \mathrm{~min}$ at $8000 \mathrm{rpm}$ after being homogenized for $2 \mathrm{~min}$ with $20 \mathrm{~mL}$ of the solvent (acetonitrile, ethyl acetate and acetone). The supernatants were transferred into centrifuge tubes with 10.0 grams of sodium chloride and vortexed for $1 \mathrm{~min}$. The mixture was centrifuged for $5 \mathrm{~min}$ at $8000 \mathrm{rpm}$. $10 \mathrm{~mL}$ of the supernatant was added into the tube with $\mathrm{MgSO}_{4}(3 \mathrm{~g})$ and different amount of PSA, then vortexed for $1 \mathrm{~min}$ again for purification and good recovery. $5 \mathrm{~mL}$ of the new supernatant was filtered by organic phase membrane $(0.22 \mu \mathrm{m})$ for determination with UPLC-MS/ MS after being centrifuged for $5 \mathrm{~min}$ at $8000 \mathrm{rpm}$ again. Blank samples and quality control samples (spiked standard) pretreated by the same method were associated with the samples to verify the operation and guarantee the results.

\section{Difenoconazole determination}

UPLC-MS/MS was employed for the analysis of the samples. The analytes were separated on an ACQUITY UPLC ${ }^{\circledR}$ BEH C18 $(2.1 \mathrm{~mm} \times 100 \mathrm{~mm}, 1.7 \mu \mathrm{m})$ column at $35^{\circ} \mathrm{C}$. The mobile phase consisted of acetonitrile (A) and water (B). The initial mobile phase composition was $10 \%$ A for $0.1 \mathrm{~min}$, following by a linear gradient to $90 \%$ A up to $3 \mathrm{~min}$, and then held for $2 \mathrm{~min}$ at $90 \%$ A. A linear gradient went down to $10 \%$ A to $5.2 \mathrm{~min}$, and then held for $1.8 \mathrm{~min}$ at $10 \% \mathrm{~A}$. The flow rate used was 0.25 $\mathrm{mL} \min ^{-1}$. Only $5 \mu \mathrm{L}$ of the samples was injected during the experiments.

The UPLC system was connected to MS/MS, as mentioned above. The instrument was operated in the positive electrospray mode $\left(\mathrm{ESI}^{+}\right)$using multiple reaction monitoring (MRM) with two mass transitions where both of the target analytes yielded $[\mathrm{M}+\mathrm{H}]^{+}$precursor ions. The more intense of the two mass transitions was used for quantitation, while the other was employed for confirmation. The basic MS source conditions were as follows: ion spray voltage (IS): $5500 \mathrm{~V}$; source temperature: $650{ }^{\circ} \mathrm{C}$; atomizing air (GS1): 55 psi, auxiliary heater (GS2): 55 psi, curtain gas (CUR): 20 psi, collision gas (CAD): 6 psi, and entrance voltage (EP): $10 \mathrm{~V}$. The Analyst 1.6.2 software platform enables instrument control and data processing for $\mathrm{AB}$ Sciex mass spectrometry systems.

\section{Detection method validation}

The method was evaluated in terms of its linearity, accuracy, precision and sensitivity, including limit of detection (LOD) and limit of quantification (LOQ). Blank samples were selected for validation purposes. Linear calibration curves were constructed by plotting the targeted pesticide peak areas obtained against the concentrations ${ }^{22}$. The standard solution was prepared by diluting the stock solution with the extract solution used for the blank samples. Linearity was assessed by the determination coefficient $\left(R^{2}\right)$ calculated from five-point $(0.005,0.01,0.020$, $0.030,0.050 \mu \mathrm{g} \mathrm{mL} \mathrm{m}^{-1}$ ) calibration curves. The accuracy of the method, represented by the spiked recoveries and their RSD, was evaluated by carrying out three consecutive extractions $(n=$ 3 ) of spiked samples at three concentration levels $(0.01,0.05$, and $0.10 \mathrm{mg} \mathrm{kg}^{-1}$ ) in the blank sample extraction solution. The LOD was determined using a signal-to-noise ratio of 3 and the LOQ was obtained by a series of spiked samples, which provided satisfactory recoveries (70-120\%) with relative standard deviations (RSDs) less than $20 \%$ in the relatively lowest spiked concentration. ${ }^{23}$

\section{Results and discussions}

\section{Sample extraction and purification procedures}

To choose the optimum extraction solvent, ethyl acetate, acetone and acetonitrile were compared in the extraction. The extractions that were carried out using ethyl acetate showed poor recovery, mostly from $60 \%$ to $80 \%$. The samples extracted with acetone showed more impurities, which would interfere with the test results and the analytical equipment. Difenoconazole was readily soluble in acetonitrile, which gave satisfactory recoveries between $90 \%$ and $105 \%$ with good RSDs $(<5 \%)$. Therefore, acetonitrile was used for the sample extraction. QuEChERS technique ${ }^{24}$ employing dispersive-solid phase extraction for purification ${ }^{25,26}$ is a fast and easy extraction method. This approach is very flexible and serves as a template for modification depending on the analyte properties, composition, equipment, and analytical techniques available in the lab. ${ }^{27}$ In this research, different amounts of PSA (50, 80, 100, 130 and $150 \mathrm{mg}$ ) were compared for the optimization, which showed that the recovery increased with the increase of the amount of PSA initially and reached a maximum when the PSA amount was $100 \mathrm{mg}$. It did not improve any further when the amount of the PSA increased, thus, the quantity of the PSA was determined.

Pesticide residue analysis mainly involves sample preparation (extraction and clean up) and instrumental analysis. Excellent sample preparation should be rapid, simple, cheap, and environmentally friendly. ${ }^{28}$ Well-matched polarity between the extraction solvent and the analyte is beneficial to improve the process efficiency and minimize interference. Acetonitrile, which is often used for the extraction of pesticides in agro-food for the low absorbance of pigments, fats and proteins, also showed advantages in environmental detection. Optimization for the amount of PSA lowered the cost of detection and all of them made the extraction and purification available.

\section{Optimization of instrumental parameters}

The standard solution was used to find the best determination parameters and develop a good analytical method. Prior to the analysis of difenoconazole residue in samples via LC/ESI-MS/MS, the target compounds prepared in pure solvent were monitored in the scan range of $100-500 \mathrm{~m} / \mathrm{z}$ to produce the best spectrum of the positively charged precursor ions $\left(406[\mathrm{M}+\mathrm{H}]^{+}\right)$. 

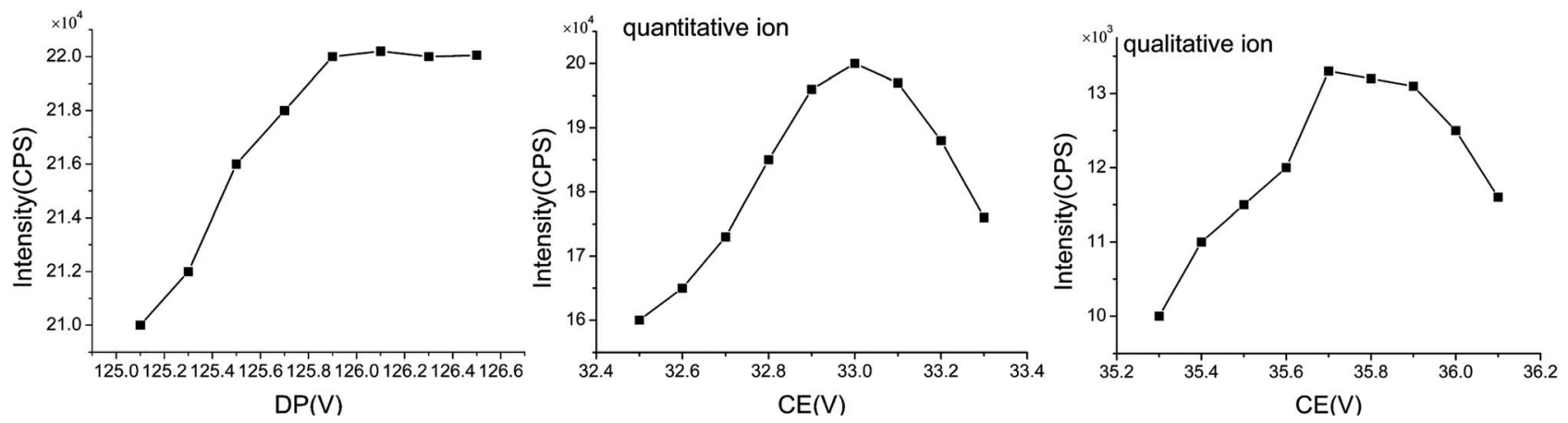

Fig. 3 The optimization of the DP and CE.

Difenoconazole showed the highest intensity at 251.00 and $337.00 \mathrm{~m} / z$, and these peaks correspond to protonation $\left([\mathbf{M}+\mathbf{H}]^{+}\right)$.

Furthermore, to obtain higher intensity, the declustering potential voltage (DP) and collision energy (CE) for collisioninduced dissociation of the precursor ions were optimized to provide better sensitivity and fragmentation (Fig. 3). The DP was adjusted with the CE fixed at $20 \mathrm{~V}$. When the DP was $125.9 \mathrm{~V}$, the ion intensity and sensitivity were the highest. Then, the DP was fixed at $125.9 \mathrm{~V}$ and the $\mathrm{CE}$ was adjusted to obtain the maximum ion intensity. The quantitative and qualitative ions had the greatest ion intensity when the CEs were $32.98 \mathrm{~V}$ and $35.70 \mathrm{~V}$, respectively.

\section{Detection method validation}

Accuracy, precision and sensitivity of the method. Ion peaks with proper retention time, good peak shape, high resolution and response can be obtained after optimization. Chromatograms of the total and quantitative ion flows of the samples are shown in Fig. 4.

Linearity was observed from 0.005 to $0.050 \mu \mathrm{g} \mathrm{mL}^{-1}$ and its correlation coefficient was 0.9967 , which supplied a wide range for the detection. The recoveries at three concentration levels (0.01, 0.05 and $0.1 \mathrm{mg} \mathrm{kg}^{-1}$ ) and their relative standard deviations (RSD, \%) were employed to evaluate the accuracy of the method. The recoveries of the pesticide in soil samples were
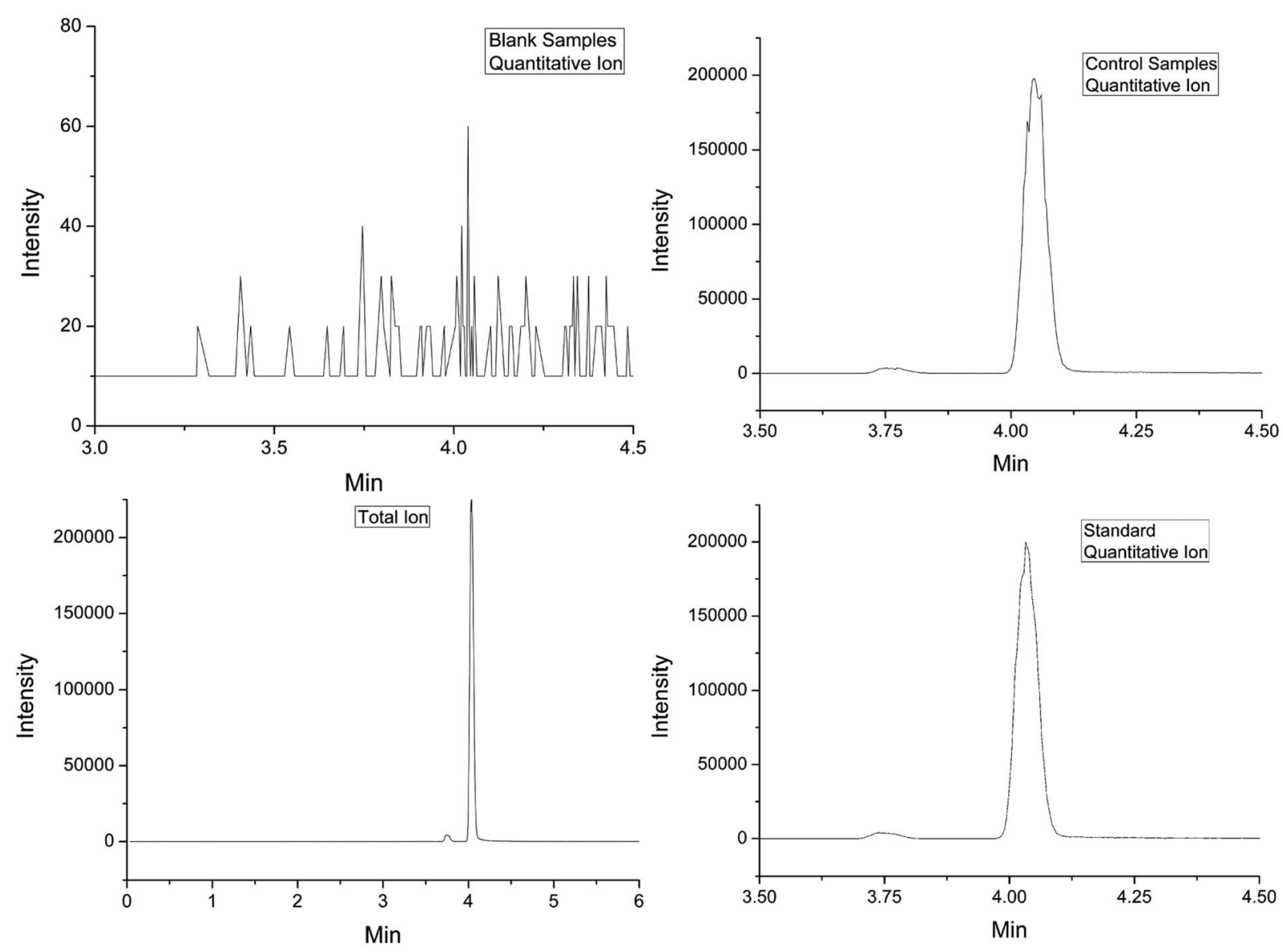

Fig. 4 The ion flow chromatogram of the samples. 
Table 1 Recoveries and RSD of difenoconazole in spiked samples $(n=3)$

\begin{tabular}{|c|c|c|c|c|}
\hline \multirow[b]{2}{*}{$\begin{array}{l}\text { Addition concentration } \\
\left(\mathrm{mg} \mathrm{kg}^{-1}\right)\end{array}$} & \multicolumn{2}{|l|}{ Soil } & \multicolumn{2}{|l|}{ Water } \\
\hline & $\begin{array}{l}\text { Recovery } \\
(\%)\end{array}$ & $\begin{array}{l}\text { RSD } \\
(\%)\end{array}$ & $\begin{array}{l}\text { Recovery } \\
(\%)\end{array}$ & $\begin{array}{l}\text { RSD } \\
(\%)\end{array}$ \\
\hline 0.01 & 104.49 & 2.68 & 98.07 & 4.74 \\
\hline 0.05 & 104.16 & 3.11 & 98.11 & 3.17 \\
\hline 0.1 & 90.29 & 2.39 & 93.75 & 3.12 \\
\hline
\end{tabular}

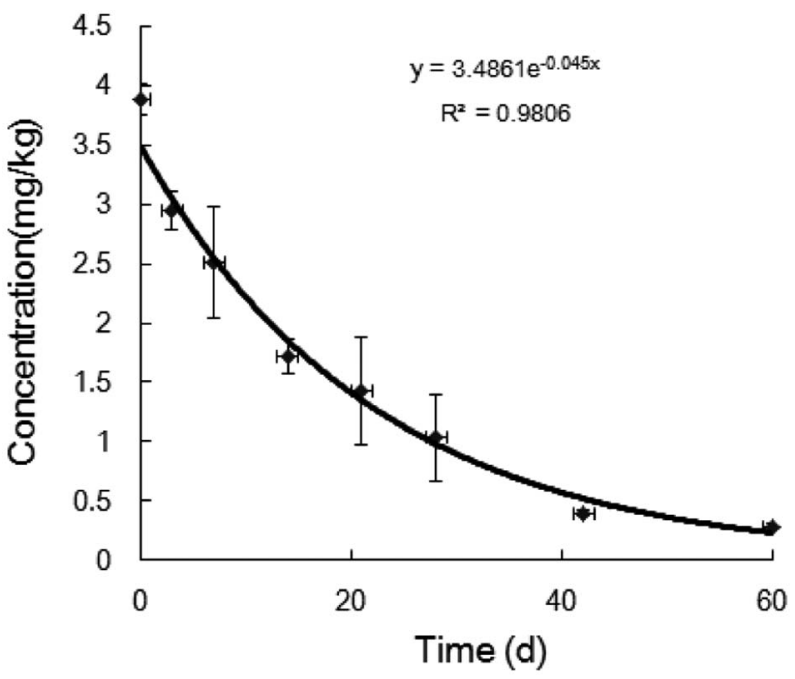

Fig. 5 The degradation of the difenoconazole in the soil.

from $90 \%$ to $105 \%$ with RSDs less than $5 \%$, which are slightly inferior to those for the water samples for complex pretreatments and matrix (Table 1). LOD and LOQ were employed to evaluate the sensitivity of the method, giving values of 0.002 and $0.035 \mathrm{mg} \mathrm{kg}^{-1}$, respectively. The results demonstrated that the accuracy of the method totally met the requirements of the pesticide detection, not only the accuracy but also the sensitivity.
Difenoconazole degradation in soil. The residue dynamics of difenoconazole in the soil was researched according to the experiment design and the results are shown in Fig. 5. The halflife was about 15.4 days and about $10 \%$ of the residue was still left in the soil 40 days later, which showed that the degradation process in the soil was relatively slow. Notably, pesticide residue in farmland would be accumulated in the soil, especially in the year-round agriculture system used in tropical area. Furthermore, 6.0, the $\mathrm{pH}$ of the soil, slowed the degradation of the residue because difenoconazole is more stable in acid media. Thus, the residue would be remarkably accumulated in the soil. Accumulated pesticide would affect the soil ecosystem to a certain extent, especially the microbiology, which is one of the most important aspects of the soil ecology. Thus, investigating the pesticide behavior in soil is significant to assess its effects on the soil health and ecology system.

Difenoconazole transportation in the soil. Transportation is another important behavior of pesticides, from the plant to the soil or from the soil to the water, which is also critical to the safety of the environment and the ecosystem. Difenoconazole concentrations in the water samples from the settlement and transportation in different locations and soil depths are shown in Fig. 6. After the first rain, in the soil under the crown at the depth of $10 \mathrm{~cm}$, the difenoconazole concentration was highest, up to $0.32 \mu \mathrm{g} \mathrm{mL} \mathrm{m}^{-1}$. The concentration declined with the increase of the depth and there was no pesticide left at the depth of $30 \mathrm{~cm}$. In the shallow layer of the soil, the concentration declined with the rainfall incidences, however, it was contrary in the deep layer of the soil. After four incidences of rainfall, the residue at the depths of 10 and $30 \mathrm{~cm}$ was about 0.042 and $0.041 \mu \mathrm{g} \mathrm{mL} \mathrm{L}^{-1}$, respectively, in the collected water. Compared with them, the samples outside the crown had much lower residues after the first rain. The concentration of the residue at the depth of $10 \mathrm{~cm}$ and $30 \mathrm{~cm}$ was 0.031 and $0 \mu \mathrm{g}$ $\mathrm{mL}^{-1}$, respectively. The same trend was also observed with the concentration in the samples under the crown. Its environmental behaviors are closely related with the conditions in the farmland, plants, microbes and weather, which are the primary factors affecting the behaviors. Thus, the results from the
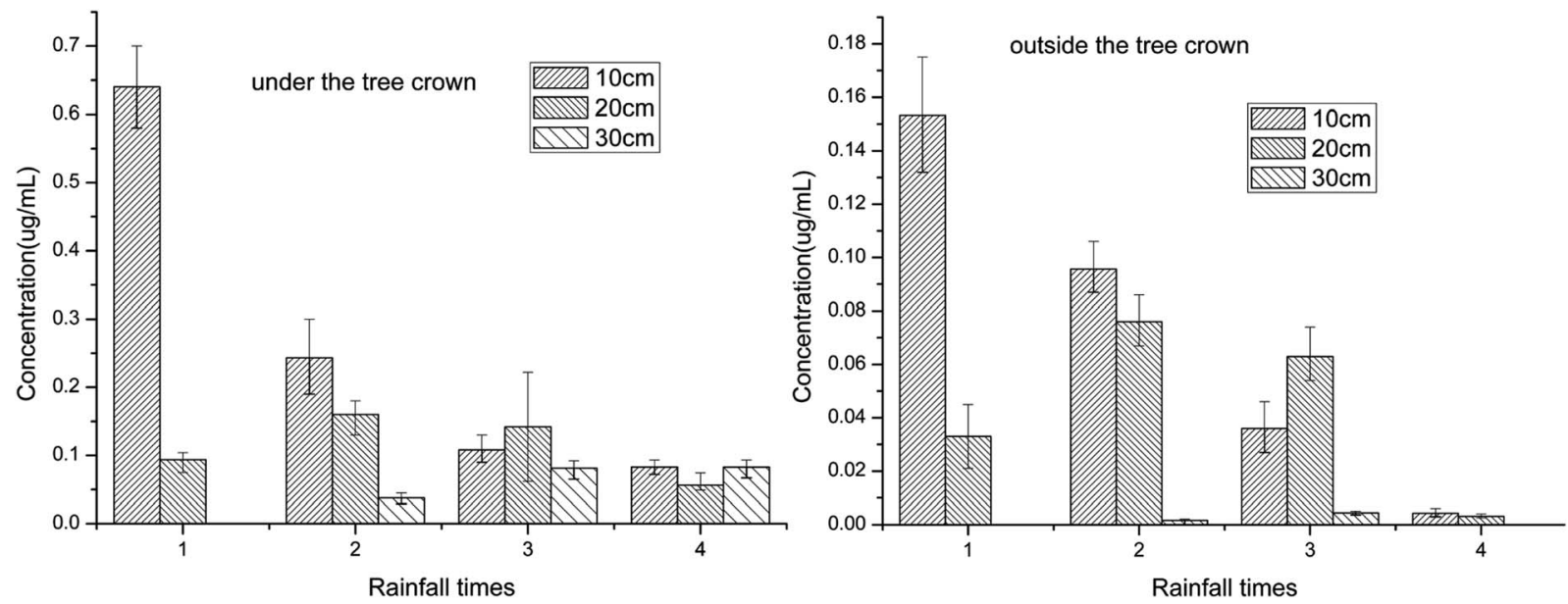

Fig. 6 The difenoconazole diffusion to the water. 
experiments were mostly in accordance with the real situation. In this experiment, the soil was sandy latosol, which is beneficial to the transportation of the residue, especially in tropical areas because it always rains heavily in summer. It has a significant influence on the residue behavior of the pesticide. The rainfall seriously affects the farmland ecosystem, which would not be reflected in the labs.

Reports $^{\mathbf{1 2 - 1 4}}$ about the behavior of difenoconazole in the field are mainly focused on its degradation, which is closely related to the environmental conditions. According to the $\mathrm{pH}$ value and type of soil, its half-life in the field and soil cultivated in the labs was 5-10 days while it was about 150-300 days under experimental conditions. The process of its degradation and transportation were the results of the pesticide-environment interactions. Soil and water are the most fundamental parts of the ecosystem, which would affect most of the organisms in the ecosystem. Therefore, the sedimentation and transportation characteristics of difenoconazole are critical in the farmland ecosystem. The results from the novel experiments proved that the difenoconazole could transport in the field environment, from the soil to the water, and probably further into the river, which would broaden the effects in the farmland and even in aquatic organisms. Perhaps it is also an important cause of damage to biodiversity. Therefore, to scientifically estimate the behaviors of the pesticides is important for ecological health.

\section{Conclusions}

Pesticide behavior is extensively influenced by the complex environmental factors in nature and simulation experiments in the lab cannot reflect their actual activity because of the great differences between nature and the lab. Pesticide behaviors are the foundation of assessing their influence on ecology and to obtain the actual behavior of the difenoconazole, a novel experiment in the field was designed. Initially, after optimization, a credible detection method was developed for the difenoconazole residue, and then the water and soil were sampled in situ without interference. Finally, the degradation and transportation characteristics were investigated using the novel designed methods.

The half-life of difenoconazole was about 15.4 days, which was in the middle level of fungicides' degradation. There was sedimentation in the soil with the heavy rain, which transported difenoconazole not only from shallow to deep layer but also in the horizontal direction. The area of residue transportation expanded gradually from the soil under the crown to outside with the rainfall. The results showed that the residue was transported by the rain, which enhanced the threat to the farm ecosystem. It is probable that the pesticide residues will be finally detected in the river for the slater of the fungicide, which may be a great threat to the whole ecosystem. Thus, the novel method could be scientific and reasonable for assessing the potential effects of pesticides on ecology.

\section{Conflicts of interest}

There are no conflicts to declare.

\section{Acknowledgements}

This work was supported by the Natural Science Fund of Hainan Province and Technique Research for Youths in 2018 (Isolation and Identification of Active Compounds in Agricultural Waste, Piper nigrum L. Tendril and Evaluation of Their Bioactivities), the Fundamental Scientific Research Funds for Chinese Academy of Tropical Agricultural Sciences (1630042014006 and 1630082016003) and Integrated Demonstration of Efficient Cyclic Utilization on the Waste of Edible Fungi ([2016]2547).

\section{References}

1 M. S. Hamada, Y. N. N. Yin and Z. H. Ma, Sensitivity to iprodione, difenoconazole and fludioxonil of Rhizoctonia cerealis isolates collected from wheat in China, Crop Prot., 2011, 30, 1028-1033.

2 Z. H. Wang, T. Yang, D. M. Qin, Y. Gong and Y. Ji, Determination and dynamics of difenoconazole residues in Chinese cabbage and soil, Chin. Chem. Lett., 2008, 19, 969972.

3 Z. Q. Kong, F. S. Dong, J. Xu, X. G. Liu, C. P. Zhang, J. Li, Y. B. Li, X. Chen, W. L. Shan and Y. Q. Zheng, Determination of difenoconazole residue in tomato during home canning by UPLC-MS-MS, Food Control, 2012, 23, 542-546.

4 Z. Y. Zhang, W. Jiang, Q. Jian, W. C. Song, Z. T. Zheng, D. L. Wang and X. J. Liu, Residues and dissipation kinetics of triazole fungicides difenoconazole and propiconazole in wheat and soil in Chinese fields, Food Chem., 2015, 168, 396-403.

5 R. Stepán, J. Tichá, J. Hajslová, T. Kovalczuk and V. Kocourek, Baby food production chain: pesticide residues in fresh apples and products, Food Addit. Contam., 2005, 22, 1231-1242.

6 F. Sanchez-Bayo and R. V. Hyne, Comparison of environmental risks of pesticides between tropical and nontropical regions, Integr. Environ. Assess. Manage., 2011, 7, 577-586.

7 S. E. Lewis, D. M. Silburn, R. S. Kookana and M. Shaw, Pesticide behavior, fate, and effects in the tropics: an overview of the current state of knowledge, J. Agric. Food Chem., 2016, 64, 3917-3924.

8 M. A. Daam and P. J. Van den Brink, Implications of differences between temperate and tropical freshwater ecosystems for the ecological risk assessment of pesticides, Ecotoxicology, 2010, 19, 24-37.

9 P. Carriquiriborde, P. Mirabella, A. Waichman, K. Solomon, P. J. Van den Brink and S. Maund, Aquatic risk assessment of pesticides in Latin America, Integr. Environ. Assess. Manage., 2014, 10, 539-542.

10 N. J. Diepens, S. Pfennig, P. J. Van den Brink, J. S. Gunnarsson, C. Ruepert and L. E. Castillo, Effect of pesticides used in banana and pineapple plantations on aquatic ecosystems in Costa Rica, J. Exp. Biol., 2014, 35, 73-84. 
11 M. Pistón, I. Machado, C. S. Branco, V. Cesio, H. Heinzen, D. Ribeiro, et al., Infusion, decoction and hydroalcoholic extracts of leaves from artichoke (Cynara cardunculus L. subsp. cardunculus) are effective scavengers of physiologically relevant ROS and RNS, Food Res. Int., 2014, 60, 150-156.

12 C. Chu, Z. Wang, D. Qin, Y. Gong and Y. Ji, Study on determination and dynamics of difenoconazole residues in celery and soil, Sci. Sin.: Chim., 2011, 41(1), 129-135.

13 M. He, C. Jia, E. Zhao, L. Chen, P. Yu, J. Jing and Y. Zheng, Concentrations and dissipation of difenoconazole and fluxapyroxad residues in apples and soil determined by ultrahigh-performance liquid chromatography electrospray ionization tandem mass spectrometry[J], Environ. Sci. Pollut. Res., 2016, 23(6), 5618-5626.

14 J. Li, F. Dong, Y. Cheng, X. Liu, J. Xu, Y. Li and Y. Zheng, Simultaneous enantioselective determination of triazole fungicide difenoconazole and its main chiral metabolite in vegetables and soil by normal-phase high-performance liquid chromatography, Anal. Bioanal. Chem., 2012, 404(67), 2017-2031.

15 L. Maltby, N. Blake, T. C. M. Brock and P. J. Van den Brink, Insecticide species sensitivity distributions: importance of test species selection and relevance to aquatic ecosystems, Environ. Toxicol. Chem., 2005, 24, 379-388.

16 K. W. Kwok, K. M. Leung, G. S. Lui, V. K. Chu, P. K. Lam, D. Morritt and M. Crane, Comparison of tropical and temperate freshwater animal species' acute sensitivities to chemicals: implications for deriving safe extrapolation factors, Integr. Environ. Assess. Manage., 2007, 3, 49-67.

17 A. Rico, A. V. Waichman, R. Geber-Corrêa and P. J. Van den Brink, Effects of malathion and carbendazim on Amazonian freshwater organisms: comparison of tropical and temperate species sensitivity distributions, Ecotoxicology, 2011, 20, 625-634.

18 A. M. Filho, F. N. D. Santos and P. A. D. P. Pereir, Development, validation and application of a methodology based on solid-phase micro extraction followed by gas chromatography coupled to mass spectrometry (SPME/GCMS) for the determination of pesticide residues in mangoes, Talanta, 2010, 81, 346-354.

19 S. Hingmire, D. P. Oulkar, S. C. Utture, T. P. A. Shabeer and K. Banerjee, Residue analysis of fipronil and difenoconazole in okra by liquid chromatography tandem mass spectrometry and their food safety evaluation, Food Chem., 2015, 176, 145-151.
20 Y. Fu, T. Yang, J. Zhao, L. Zhang, R. X. Chen and Y. L. Wu, Determination of eight pesticides in Lycium barbarum by LC-MS_MS and dietary risk assessment, Food Chem., 2017, 218, 192-198.

$21 \mathrm{M}$. Reuveni and D. Sheglov, Effects of azoxystrobin, difenoconazole, polyoxin B (polar) and trifloxystrobin on germination and growth of Alternaria and decay in red delicious apple fruit, Crop Prot., 2002, 21, 951-955.

22 European Commission Directorate-General for Health and Food Safety. Guidance document on analytical quality control and method validation procedures for pesticides residues analysis in food and feed (SANTE 11945/2015). 2015.

23 P. Sivaperumal, P. Anand and L. Riddhi, Rapid determination of pesticide residues in fruits and vegetables, using ultra-high-performance liquid chromatography/time-of-flight mass spectrometry, Food Chem., 2015, 168, 356-365.

24 S.-K. Cho, A. M. Abd El-Aty, Md. Musfiqur Rahman, J.-H. Choi and J.-H. Shim, Simultaneous multidetermination and transfer of eight pesticide residues from green tea leaves to infusion using gas chromatography, Food Chem., 2014, 165, 532-539.

25 M. Anastassiades, S. J. Lehotay, D. Stajnbaher and F. J. Schenck, Fast and easy multiresidue method employing acetonitrile extraction/partitioning and "dispersive solid-phase extraction" for the determination of pesticide residues in produce, J. AOAC Int., 2003, 86, 412-431.

26 J. A. Ferreira, J. M. S. Ferreira, V. Talamini, J. D. F. Facco, T. M. Rizzetti, O. D. Prestes, M. B. Adaime, R. Zanella and C. B. G. Bottoli, Determination of pesticides in coconut (Cocos nucifera Linn.) water and pulp using modified QuEChERS and LC-MS/MS, Food Chem., 2016, 213, 616-624.

27 I. Machado, N. Gérez, M. Pistón, H. Heinzen and M. V. Cesio, Determination of pesticide residues in artichoke leaves and fruits by GC-MS and LC-MS/MS using the same QuEChERS procedure, Food Chem., 2017, 227, 227-236.

28 M. M. Rahman, W. Farha, A. M. A. El-Aty, Md. H. Kabir, S. J. Im, D. Jung, J. H. Choi, S. W. Kim, Y. W. Son, C. H. Kwon, H. C. Shin and J. H. Shim, Dynamic behavior and residual pattern of thiamethoxam and its metabolite clothianidin in Swiss chard using liquid chromatographytandem mass spectrometry, Food Chem., 2015, 174, 248-255. 